\title{
Droplet Coalescence is Initiated by Thermal Motion
}

\author{
Sreehari Perumanath, ${ }^{1, *}$ Matthew K. Borg, ${ }^{1}$ Mykyta V. Chubynsky, ${ }^{2}$ James E. Sprittles, ${ }^{2}$ and Jason M. Reese ${ }^{1, \dagger}$ \\ ${ }^{1}$ School of Engineering, University of Edinburgh, Edinburgh EH9 3FB, United Kingdom \\ ${ }^{2}$ Mathematics Institute, University of Warwick, Coventry CV4 7AL, United Kingdom
}

(Received 24 May 2018; revised manuscript received 11 September 2018; published 13 March 2019)

\begin{abstract}
The classical notion of the coalescence of two droplets of the same radius $R$ is that surface tension drives an initially singular flow. In this Letter we show, using molecular dynamics simulations of coalescing water nanodroplets, that after single or multiple bridges form due to the presence of thermal capillary waves, the bridge growth commences in a thermal regime. Here, the bridges expand linearly in time much faster than the viscous-capillary speed due to collective molecular jumps near the bridge fronts. Transition to the classical hydrodynamic regime only occurs once the bridge radius exceeds a thermal length scale $l_{T} \sim \sqrt{R}$.
\end{abstract}

DOI: 10.1103/PhysRevLett.122.104501

Understanding the dynamics of coalescing liquid droplets is crucial to diverse natural and man-made processes. These include storm cloud formation [1], thermal management of MEMS devices [2,3], coating technologies [4], water harvesting through condensation [5] etc. We wish to understand two stages in the coalescence of two droplets: (i) how the droplets first join, and (ii) how the formed liquid bridge subsequently grows.

Thermal capillary waves are created in the interplay between the thermal motion of constituent molecules, which act to distort the interface between two phases, and surface tension, which acts to suppress increases in interfacial area. Such fluctuations on droplet surfaces can influence both stages of coalescence, potentially manifesting themselves at much larger scales than their amplitude $\left(\sigma \sim \sqrt{k_{B} T / \gamma}\right.$, where $\gamma$ is the surface tension), as we discuss below. In the case of liquid jet breakup, the importance of thermal fluctuations has been uncovered through molecular dynamics (MD) studies [6], observed in experiments [7], and used to derive new scaling laws [8]. Previous studies have also considered the spontaneous growth of hydrodynamic instabilities caused by thermal fluctuations, and subsequent drainage of a fluid film between droplets to initiate coalescence [9-11]. In such cases, a thin intervening film of roughly constant width exists for a long enough time for the instabilities to grow. However, there may also be cases where the growth rate of the instability is sufficiently small that the fluctuations do not develop significantly before contact. Here we consider one such case, that of nanodroplets, and conjecture others.

Published by the American Physical Society under the terms of the Creative Commons Attribution 4.0 International license. Further distribution of this work must maintain attribution to the author(s) and the published article's title, journal citation, and DOI.
From a theoretical perspective, classical models for stage (ii) predict singularities $[12,13]$ caused by infinite free surface curvature when the droplets first meet. Much of the focus has therefore previously been on this stage, where scaling laws for the bridge evolution can be derived for different force balances of inertia, capillarity and viscosity, e.g., Ref. [14], depending on the ratio of characteristic scales to the viscous length scale $l_{v}=\mu^{2} / \rho \gamma$ (where $\mu$ is the viscosity and $\rho$ is the density). Experimentally, optical imaging techniques have provided much insight, e.g., Ref. [15], but it is only with the advent of electrical measurements that bridge radii as small as a few micrometres can be accessed [16]. Notably, however, this scale is still well above that at which the initial stages of coalescence may be expected to occur, i.e., the nanoscale. This leaves clear opportunities for further theoretical and experimental exposition.

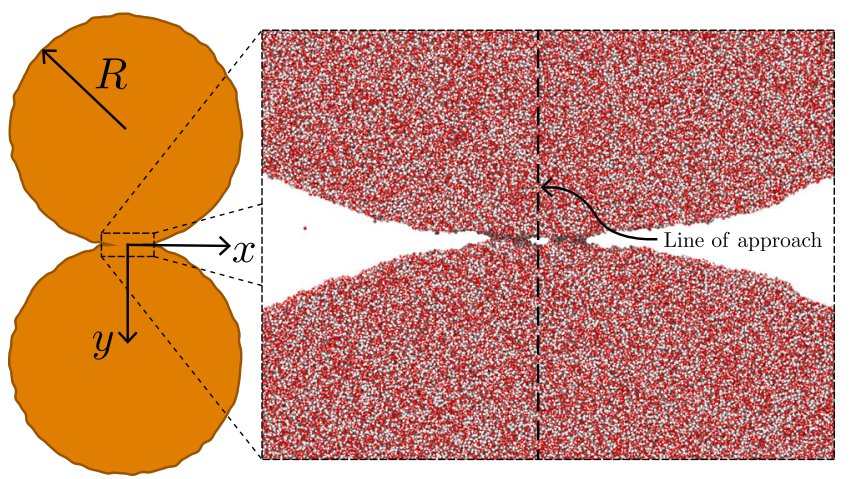

FIG. 1. The coalescence of two cylindrical droplets, each with $R=58.5 \mathrm{~nm}$ (left), and a snapshot of a close-up of the onset of coalescence in our MD simulations (right), which is characterized by multiple local bridges between the droplets caused by thermal fluctuations. In the figure, red denotes oxygen and white denotes hydrogen atoms. 
In this Letter we show that (a) the contact between coalescing nanodroplets is initiated by thermal fluctuations on the surfaces, with no evidence of an instability, and (b) after contact, a new regime of bridge evolution occurs until the bridge reaches a length $l_{T}$ from the collision axis. The dynamics of bridge growth within this length, which we denote the thermal length scale, is so fast that it is practically inaccessible to experimental measurements on pure liquids, even using recent electrical methods. At present, we can only investigate these small spatiotemporal scales using high-fidelity molecular simulations. This new nonclassical thermal regime of droplet coalescence, revealed by our simulations, precedes the viscous regime and supplants classical considerations of singularities. Conventional hydrodynamic models are only applicable in the later stages of coalescence.

MD simulations.-We study the early stages of the coalescence of two free water droplets by performing unconventionally large and computationally expensive MD simulations [17] using the LAMMPS software [18]. We simulate droplet coalescence in close-to-vacuum conditions in order to focus on the effects of thermal fluctuations in the absence of film drainage effects. We choose liquid water in the present study because of its wide practical applications $[5,19,20]$. A schematic is provided in Fig. 1. The rigid four-site model of water TIP4P/2005 $[21,22]$ is used in all the major simulations; a different model, the one-site $\mathrm{mW}$ model [23], was also considered in order to study the influence of the interatomic potentials on the results (please see the Supplemental Material (SM) for details [24]). We investigate both spherical (3D) and cylindrical (quasi 2D) droplets of identical radius $R$ by placing two droplets with a slight separation and then bringing them together with a small velocity, similar to experimental analyses. Previous studies have shown that cylindrical discs can qualitatively represent the coalescence of spheres [25,26], and an analytical solution for the bridge growth in $2 \mathrm{D}$ at its early stages [13] has been shown to be asymptotically equivalent to that for 3D coalescence [12]. Most of the investigations in this Letter are therefore on cylindrical droplets (with an axial length $L$ ), because this enables larger droplets and more realizations to be simulated. Further details of the simulations are provided in the SM.

Stochastic coalescence.-The shape of a free droplet can be assumed to comprise a mean profile (i.e., a sphere) and a fluctuating part, which arises due to the presence of thermal capillary waves on the surface. Our MD simulations show that these spatiotemporal fluctuations at the interface make the onset of coalescence a stochastic process. On a free droplet surface, the local thermal fluctuations about the mean interface profile have a standard deviation that can be determined using the theory of thermal capillary waves [27-31] (see SM). If we define $x$ along the normal to the collision axis (or line of approach, see Fig. 1), then the

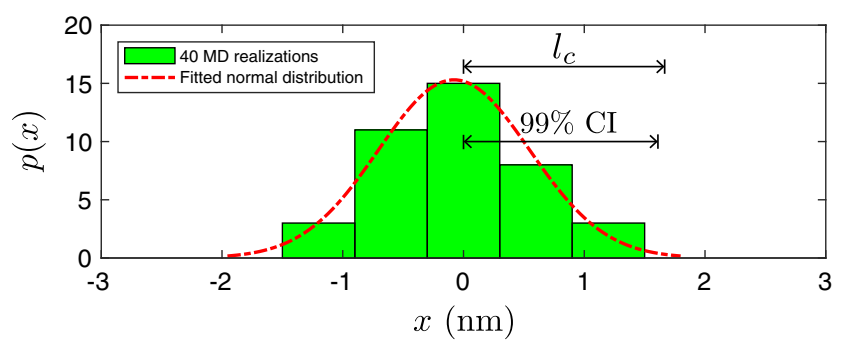

FIG. 2. The distribution along $x$ of the coalescence points for two cylindrical droplets of radii $R=11.1 \mathrm{~nm}$, obtained from 40 independent MD realizations.

locations where coalescence initiates are observed to be distributed along $x$ with the most probable location at $x=0$ (see Fig. 2). We obtain this distribution from 40 independent MD realizations of the coalescence of two cylindrical droplets with $R=11.1 \mathrm{~nm}$, and determine the width of the spread of the contact points [99\% confidence interval (CI)] by fitting a Gaussian function. Based on the relative fluctuation of neighboring points on the surface, assuming that the fluctuations on one droplet are not affected by the presence of the other, and there are no instabilities, we also derive an order-of-magnitude estimate of this width (see SM):

$$
l_{c} \approx\left(\frac{k_{B} T}{\gamma}\right)^{1 / 4} R^{1 / 2}
$$

In Fig. $2, l_{c}$ is seen to be comparable to the $99 \%$ CI of the distribution of contact points from our MD simulations, within a prefactor of order unity. In the region $|x|<l_{c}$, we observe that coalescence initiates as if the fluctuations of two independent droplets meet from opposite sides. Multiple bridges within $l_{c}$ are also possible (see Fig. 1).

Physical mechanism.-We investigate the mechanism of bridge growth after the first contact by observing the dynamics of molecules close to a bridge front. During the initial stages, bridge fronts propagate by means of collective molecular jumps [Fig. 3(a)], in contrast to the ordered circulatory bridging flow from under a bridge that is characteristic of the classical viscous and inertial regimes that occur later [Fig. 3(b)].

We classify this initial stage as the thermal regime, where a bridge grows due to molecules moving directly across the intervening gap between the droplets, caused by the proximity of the confronting surfaces of the droplets coupled with the effect of thermal motion. After first contact at a molecular scale, the surfaces of the droplets appear to be drawn into each other across the gaps on either side of a bridge (see video in SM). As more molecules from both droplets populate this gap, the outer and inner bridge fronts (in the case of multiple contacts) propagate until either two bridge fronts meet (in the case of inner bridges) or the outer bridge fronts grow and the gap eventually becomes too large for thermal fluctuations to dominate bridge growth. The hydrodynamic mechanism takes over 


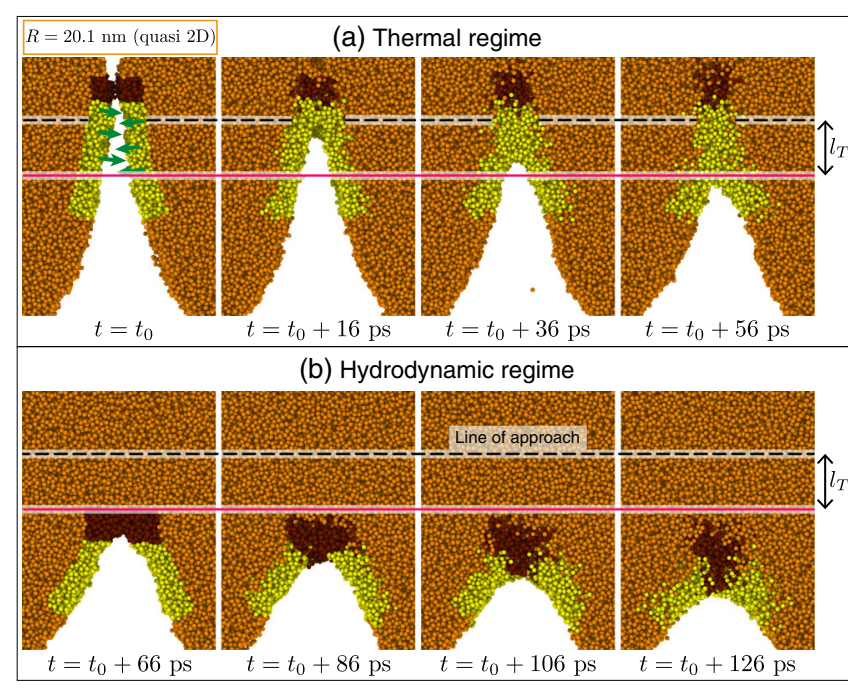

FIG. 3. MD simulation snapshots (quasi 2D; $R=20.1 \mathrm{~nm}$ ) from a reference time $t_{0}$ when coalescence just begins, showing bridge growth in (a) the thermal regime by collective molecular jumps from both droplets (i.e., due to the yellow colored atoms), and (b) the hydrodynamic regime, which is the classical bridging flow from under the bridge (i.e., due to the maroon colored atoms). Only the oxygen atoms are shown, and are colored for illustrative purposes only.

when the outer bridge fronts have grown past a droplet radius-dependent length scale $l_{T}$, which we quantify below.

We find that, in the thermal regime, the collective motion of the molecules strongly affects bridge growth in time, described by $r_{\mathrm{b}}(t)$, which is measured from the line of approach. Figure 4 shows three instances of a single bridge formed between the droplet interfaces (i.e., the equimolar line where the local density is the average of the liquid and vapor densities) immediately after coalescence has begun:

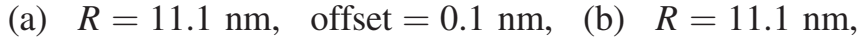
offset $=-1 \mathrm{~nm}$ and (c) $R=58.5 \mathrm{~nm}$, offset $=-4 \mathrm{~nm}$, where the offset is the distance between the line of
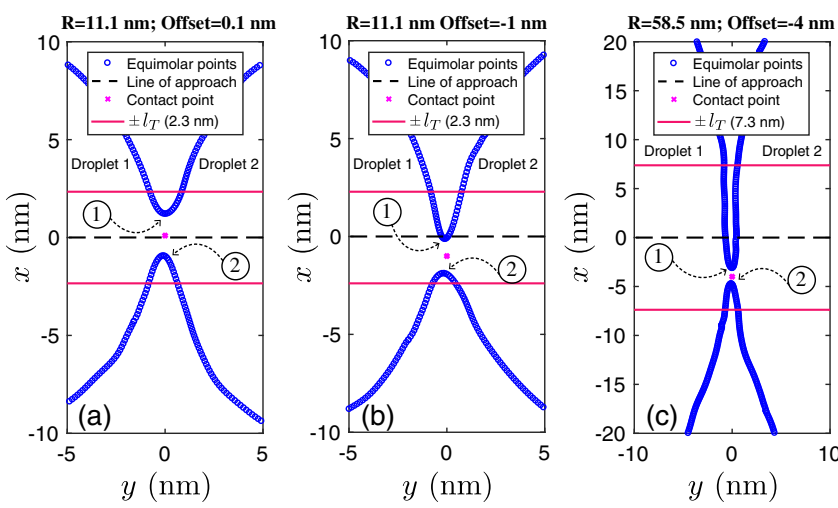

FIG. 4. Droplet interfaces for $R=11.1 \mathrm{~nm}$ and $58.5 \mathrm{~nm}$ quasi $2 \mathrm{D}$ droplets immediately after the onset of coalescence. The line of approach connects the centers of mass of each droplets. approach and the initial contact point. Figure 5 shows the corresponding bridge growths in time.

Bridge growth in its early stages is observed to be linear in time. From our results, we determine the thermal length scale $l_{T}$ as the length over which the bridge grows linearly as a result of collective molecular jumps (see SM). Interestingly, we note that $l_{T} \approx 2 l_{c}$ (i.e., $l_{T} \sim l_{c}$ ) for both spherical and cylindrical droplets, which is reasonable since both lengths are defined by thermal mechanisms. Remarkably, for spherical droplets with $R \sim 1 \mathrm{~mm}$ (the size usually studied in experiments), assuming that the above relations are still valid, $l_{T} \sim 1 \mu \mathrm{m}$, so nanoscale effects are amplified by the high-aspect-ratio geometry of the gap between the droplets, and dominate the initial bridge formation.

We study bridge growth in the thermal regime by measuring the rate of change of total surface area during coalescence. With MD, we are able to measure the number of interface molecules over the entire surfaces of the droplets as a function of time, which is a direct indication of the surface area (see SM). This enables us to estimate the bridge growth velocity (for quasi $2 \mathrm{D}$ and $3 \mathrm{D}$ systems) in the thermal regime as

$$
v_{\mathrm{b}} \equiv \frac{d r_{\mathrm{b}}}{d t} \approx \frac{-d N / d t}{2 n_{A} \Delta Z}
$$

where $-d N / d t$ is the rate of loss of interface molecules near a bridge front of length $\Delta Z$ over which molecular jumps occur (see below), and $n_{A}$ is the number of interface molecules per unit surface area of a free droplet. For the 3D spherical case, $\Delta Z$ is the circumference of the bridge.

For quasi $2 \mathrm{D}$ systems with a single contact point between the droplets, we can track the variation of the number of interface molecules near a particular bridge front, i.e., above or below that contact point separately, and
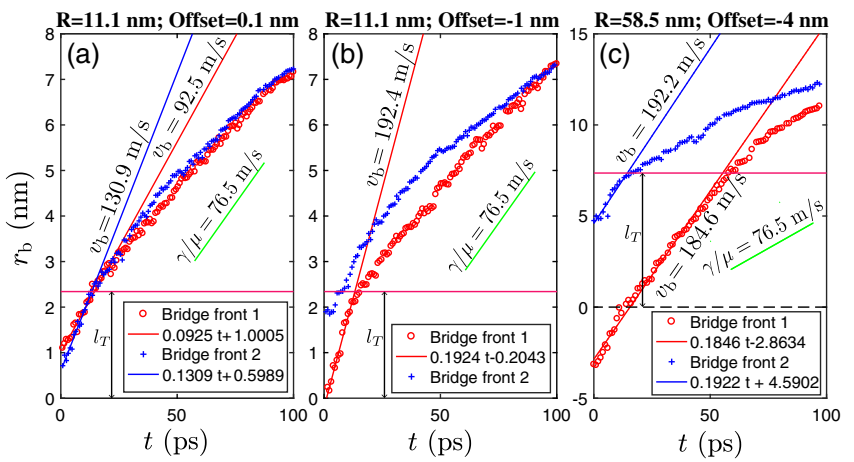

FIG. 5. Bridge growths corresponding to the cases presented in Fig. 4. Bridge front location is measured from the line of approach. Bridge growth qualitatively changes beyond $l_{T}$. No line is fit in (b) to the " + " markers within $l_{T}$, because there are not an appreciable number of data points. Note that the time here is measured from when a bridge appears in the equimolar plots; actual coalescence might have started earlier (see SM). 

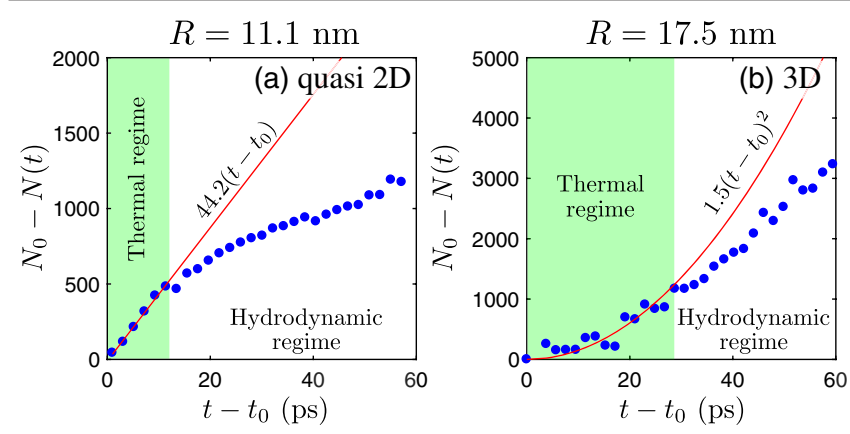

FIG. 6. The variation of the number of interface molecules $N(t)$ near a bridge front after the onset of coalescence at $t_{0}$. (a) For quasi 2D droplets, $N(t)$ is linear within the thermal regime. (b) For 3D droplets, $N(t) \sim-t^{2}$.

consequently $\Delta Z$ is the length of a single front $(L)$. In this case, we observe $N(t)$ to vary linearly in time in the thermal regime: $N_{0}-N(t)=K_{\mathrm{T}}\left(t-t_{0}\right)$, where $N_{0}$ denotes the number of interface molecules above or below the contact point before coalescence began, $N(t)$ is the instantaneous number of interface molecules above or below that contact point, $K_{\mathrm{T}} \equiv-d N / d t$, and $t_{0}$ is the time at which that contact occurred.

For instance, near the bridge front 1 in Fig. 4(b), with a value of $-d N / d t \approx 44.2$ molecules/ps [see Fig. 6(a)], $L=4.3 \mathrm{~nm}$, and $n_{A} \approx 25$ molecules $/ \mathrm{nm}^{2}$, Eq. (2) predicts $v_{\mathrm{b}} \approx 200 \mathrm{~m} / \mathrm{s}$, which is close to the observed bridge growth velocity [Fig. 5(b)] at an early stage of coalescence, and is much greater than the viscous-capillary velocity scale $\gamma / \mu \approx 75 \mathrm{~m} / \mathrm{s}$ for water. The smaller rate of bridge growth in the case of Fig. 5(a) can be attributed to a smaller curvature at the bridge front arising from the initial contact being close to the line of approach, leading to a smaller $d N / d t$, which results in a lower velocity.

As expected, beyond the thermal regime the bridge evolution qualitatively changes and is no longer linear in time. It is also interesting to note from Figs. 5(b) and 5(c) that even if the bridge fronts are not symmetric about the line of approach in their initial stage, symmetry tends to develop at later times, as is usually assumed in classical studies.

In the coalescence of 3D spherical droplets, the region within which a contact can occur is delineated by a circle of radius $l_{c}$ centered on the line of approach. As with quasi 2D droplets, we have verified that in our 3D droplet simulations the bridge grows within the thermal regime through collective molecular jumps (see SM). We track the total number of interface molecules as a function of time, $N(t)$. In this case, $N_{0}$ signifies the total number of interface molecules before the onset of coalescence. However, in this geometry, the length over which molecular jumps can occur $\left(\Delta Z=2 \pi r_{\mathrm{b}}\right)$ increases as the bridge grows, and so the rate of loss of interface molecules $(d N / d t)$ must also increase. Since the bridge expansion in this case is also observed to be linear in time within the thermal regime (see SM), then $r_{\mathrm{b}} \sim t$ and $v_{\mathrm{b}} \sim t^{0}$, so from Eq. (2) $-d N / d t \sim t$ and consequently $N(t) \sim-t^{2}$ after the onset of coalescence [see Fig. 6(b)]. When the bridge radius expands past $l_{T}$, surface tension starts to drive the bridge front, and coalescence will proceed as usual to form a larger spherical droplet.

Discussion.-Previous theoretical analyses of coalescence have considered a singular process driven only by surface tension and resisted by a combination of viscous and/or inertial forces. In this Letter, we have found that the key driving mechanism in the initial stages is actually thermal fluctuations, and that surface tension acts to suppress this motion. The fact that molecular effects have a macroscale influence for larger droplets (i.e., $l_{T} \sim 1 \mu \mathrm{m}$ when $R \sim 1 \mathrm{~mm}$ ) calls into question the relevance of continuum simulations that go below the thermal length scale [32]. Only once $r_{\mathrm{b}} \sim l_{T}$ is reached in the coalescence does surface tension-driven bridging flow occur, which is described well by classical hydrodynamics.

In experimental studies, with a single bridge expanding radially outwards at a high speed given by Eq. (2), the thermal regime for two water droplets of radii $2 \mathrm{~mm}$ is over within a few nanoseconds; this is below the temporal resolution of the latest experiments [16]. Notably, in the electrical method employed in recent studies [16,33,34], electrostatic forces may have affected the size of the initial contact by deforming the two surfaces at a very early stage and altering the local geometry. The extent of the thermal regime may then have been affected as well.

Molecular dynamics is perhaps the only current technique that can provide insight into the role of thermal capillary waves in the coalescence of pure liquids, because of the nanoscale amplitude of these waves. A previous MD work on liquid droplet coalescence [25] failed to capture the thermal regime because of low-resolution measurements. In the present Letter, we observe that thermal fluctuations initiate coalescence before hydrodynamic instabilities get time to grow. For smaller droplets, this happens even at slow speeds of approach, since their size restricts the available fluctuation wavelengths, resulting in a slower growth rate of instability. For larger droplets colliding in a vacuum at high speeds, we expect the same coalescence mechanism (see SM).

In our theoretical analysis where we derived Eq. (1) (see SM, page 4), we have not considered the interatomic attractions between molecules in the different droplets. These may, however, be important in two ways: first, by modifying the spectrum of fluctuations of one surface when the other is close; and second, by drawing the surfaces towards each other both before and after a contact is initiated. These effects may contribute to the dependence of $v_{b}$ on the curvature of the bridge front, but the fact that the simulation results match our analysis even though we ignore them seems to suggest they are not of major importance.

We have also not considered the influence of an external fluid. In the most general theoretical model, the effect of 
van der Waals forces should be considered simultaneously with thermal fluctuations and a thin intervening fluid film in order to determine the initial location of coalescence. Coalescing droplets in oil-in-water emulsions $[11,35]$ and bubble coalescence [36] can be regarded as suitable examples, and we intend to study such systems in detail in the future.

Our framework is universal in that it is easily extended to coalescing droplets on a planar interface, and coalescing droplets of different radii (see SM, page 4). An open question is whether or not the thermal regime can be incorporated into an extended hydrodynamic framework, or whether this initial stage must be omitted from any such analysis. The collective molecular jump mechanism has parallels with the molecular kinetic theory [37] used in continuum models of wetting, and there are also similarities to interface formation or disappearance processes already applied to coalescence [38]. So there are precedents for building macroscopic models incorporating additional molecular physics.

Thermal fluctuations have already been incorporated into continuum fluid models for the breakup of liquid jets [6], by using fluctuating hydrodynamic theory [39]. Interestingly, in the liquid jet case the models have been validated experimentally using ultralow surface tension mixtures [40] in which thermal motions are visible at much larger scales. We posit that such techniques could also be capable of verifying the thermal regime in droplet coalescence that we have described in this Letter.

All data within the publication can be freely accessed from [41].

The authors are indebted to Benjamin Goddard and Andrew Parry for useful discussions and the reviewers for their constructive criticisms. The simulation results were obtained using ARCHER, the UK's national supercomputer. This research is supported by EPSRC Grants No. EP/ N016602/1, No. EP/P020887/1, No. EP/P031684/1, No. EP/R007438/1, and the Leverhulme Trust. J. M. R is supported by the Royal Academy of Engineering under the Chair in Emerging Technologies scheme.

*sreehari.dharmapalan@ed.ac.uk †jason.reese@ed.ac.uk

[1] A. Kovetz and B. Olund, J. Atmos. Sci. 26, 1060 (1969).

[2] J. B. Boreyko and C. H. Chen, Phys. Rev. Lett. 103, 2 (2009).

[3] N. Miljkovic, R. Enright, Y. Nam, K. Lopez, N. Dou, J. Sack, and E. N. Wang, Nano Lett. 13, 179 (2013).

[4] W. D. Ristenpart, P. M. McCalla, R. V. Roy, and H. A. Stone, Phys. Rev. Lett. 97, 064501 (2006).

[5] N. Miljkovic, R. Enright, and E. N. Wang, ACS Nano 6, 1776 (2012).

[6] M. Moseler and U. Landman, Science 289, 1165 (2000).
[7] Y. Hennequin, D. G. A. L. Aarts, J. H. van der Wiel, G. Wegdam, J. Eggers, H. N. W. Lekkerkerker, and D. Bonn, Phys. Rev. Lett. 97, 244502 (2006).

[8] J. Eggers, Phys. Rev. Lett. 89, 084502 (2002).

[9] A. Vrij, Discuss. Faraday Soc. 42, 23 (1966).

[10] A. Vrij and J. T. G. Overbeek, J. Am. Chem. Soc. 90, 3074 (1968).

[11] L. G. Leal, Phys. Fluids 16, 1833 (2004).

[12] J. Eggers, J. R. Lister, and H. A. Stone, J. Fluid Mech. 401, 293 (1999).

[13] R. W. Hopper, J. Fluid Mech. 213, 349 (1990).

[14] J. D. Paulsen, J. C. Burton, S. R. Nagel, S. Appathurai, M. T. Harris, and O. A. Basaran, Proc. Natl. Acad. Sci. U.S.A. 109, 6857 (2012).

[15] S. T. Thoroddsen, K. Takehara, and T. G. Etoh, J. Fluid Mech. 527, 85 (2005).

[16] J. D. Paulsen, J. C. Burton, and S. R. Nagel, Phys. Rev. Lett. 106, 114501 (2011).

[17] M. P. Allen and D. J. Tildesley, Computer Simulation of Liquids, 2nd ed. (Oxford University Press, New York, 2017).

[18] S. Plimpton, J. Comput. Phys. 117, 1 (1995).

[19] J. A. Watson, B. W. Cribb, H. M. Hu, and G. S. Watson, Biophys. J. 100, 1149 (2011).

[20] K. M. Wisdom, J. A. Watson, X. Qu, F. Liu, G. S. Watson, and C.-H. Chen, Proc. Natl. Acad. Sci. U.S.A. 110, 7992 (2013).

[21] J. L. Abascal and C. Vega, J. Chem. Phys. 123, 234505 (2005).

[22] C. Vega and E. de Miguel, J. Chem. Phys. 126, 154707 (2007).

[23] V. Molinero and E. B. Moore, J. Phys. Chem. B 113, 4008 (2009).

[24] See Supplemental Material at http://link.aps.org/ supplemental/10.1103/PhysRevLett.122.104501, which contains technical details that are complementary to this Letter. We discuss (i) the molecular dynamics simulation details and initial conditions, (ii) thermal fluctuations on liquid surfaces, (iii) the probable region of the onset of coalescence, (iv) bridge growth in the thermal regime, (v) calculations of thermal fluctuations on the surface of a thin cylinder, and (vi) the role of van der Waals interactions between the droplets. Readers are also invited to view the supplementary movie of the molecular simulations.

[25] J. C. Pothier and L. J. Lewis, Phys. Rev. B 85, 115447 (2012).

[26] J. C. Burton and P. Taborek, Phys. Rev. Lett. 98, 224502 (2007).

[27] J. S. Rowlinson and B. Widom, Molecular Theory of Capillarity (Clarendon Press, Oxford, 1982).

[28] A. Werner, F. Schmid, M. Müller, and K. Binder, Phys. Rev. E 59, 728 (1999).

[29] S. W. Sides, G. S. Grest, and M.-D. Lacasse, Phys. Rev. E 60, 6708 (1999).

[30] J. Eggers and E. Villermaux, Rep. Prog. Phys. 71, 036601 (2008).

[31] J. Lucassen, M. van den Tempel, A. Vrij, and F. Th. Hesselink, Proc. K. Ned. Akad. Wet. Ser. B Phys. Sci. 73, 109 (1970).

[32] J. E. Sprittles and Y. D. Shikhmurzaev, J. Fluid Mech. 753, 279 (2014). 
[33] S. C. Case and S. R. Nagel, Phys. Rev. Lett. 100, 084503 (2008).

[34] S. C. Case, Phys. Rev. E 79, 026307 (2009).

[35] A. K. Chesters, Trans. Inst. Chem. Eng. 69A, 259 (1991).

[36] I. U. Vakarelski, R. Manica, X. Tang, S. J. O’Shea, G. W. Stevens, F. Grieser, R. R. Dagastine, and D. Y. C. Chan, Proc. Natl. Acad. Sci. U.S.A. 107, 11177 (2010).

[37] T. D. Blake, A. Clarke, J. De Coninck, and M. J. de Ruijter, Langmuir 13, 2164 (1997).
[38] J. E. Sprittles and Y.D. Shikhmurzaev, Phys. Fluids 24, 122105 (2012).

[39] L. D. Landau and E. M. Lifshitz, Statistical Physics Part 2, Course of Theoretical Physics (Pergamon Press, Oxford, 1980), Vol. 9, pp. 86-91.

[40] D. G. A. L. Aarts, M. Schmidt, and H. N. W. Lekkerkerker, Science 304, 847 (2004).

[41] https://doi.org/10.7488/ds/2488. 